\title{
Médiévales
}

Langues, Textes, Histoire

54 | printemps 2008

Frères et sœurs

\section{Les frères et les sœurs, «parents pauvres » de la parenté}

Didier Lett

\section{(2) OpenEdition}

1 Journals

Édition électronique

URL : https://journals.openedition.org/medievales/4473

DOI : $10.4000 /$ medievales. 4473

ISSN : $1777-5892$

Éditeur

Presses universitaires de Vincennes

Édition imprimée

Date de publication : 1 juin 2008

Pagination : 5-12

ISBN : 978-2-84292-217-7

ISSN : 0751-2708

Référence électronique

Didier Lett, « Les frères et les sœurs, «parents pauvres » de la parenté », Médiévales [En ligne], 54 |

printemps 2008, mis en ligne le 10 septembre 2010, consulté le 23 avril 2022. URL : http://

journals.openedition.org/medievales/4473; DOI : https://doi.org/10.4000/medievales.4473

Ce document a été généré automatiquement le 23 avril 2022.

Tous droits réservés 


\title{
Les frères et les sœurs, « parents pauvres » de la parenté
}

\author{
Didier Lett
}

1 Les médiévistes du début du xxI siècle qui étudient la famille et la parenté inscrivent majoritairement leurs travaux dans le champ de l'anthropologie de la parenté ${ }^{1}$. Celle-ci, amorcée par le colloque de 1974, tenu à Paris, et publié trois ans plus tard, sous la direction de Georges Duby et Jacques Le Goff ${ }^{2}$, a permis de mieux comprendre les systèmes de parenté médiévale et les règles d'alliance soulignant, en particulier, l'importance de la filiation cognatique et de la parenté spirituelle. Cependant, ces travaux, fortement marqués par l'anthropologie structurale, décrivent des normes culturelles et symboliques, délaissant les relations vécues par les acteurs sociaux parfois très éloignées des liens théoriques reliant les membres d'un système de parenté reconstitué par l'historien ${ }^{3}$. Il n'est pas inutile de rappeler qu'en 1958, Claude LéviStrauss considérait que la locution «système de parenté » recouvrait deux ordres très différents de réalité. Aux côtés du système des appellations (la nomenclature théorique qui n'induit pas nécessairement une conduite à tenir), il distinguait le système des attitudes (respect ou familiarité, droit ou devoir, affection ou hostilité). Il écrivait : « le tableau des termes de parenté ne reflète pas exactement celui des attitudes familiales, et inversement. On se tromperait en croyant que dans toute société, le système de parenté constitue le medium principal par lequel se règlent les relations individuelles ${ }^{4}$.»

2 Comme le souligne Bernhard Jussen, critique à l'égard de l'historiographie française de la parenté, « la parenté comme forme d'organisation sociale a été largement surévaluée par la recherche ${ }^{5} . »$ Ce sont les échanges matériels, affectifs, symboliques qui actualisent les solidarités familiales. Maurice Godelier rappelle que, « ...dans l'immense majorité des sociétés connues, les rapports de parenté naissent de la mise en pratique, par les individus et les groupes auxquels ils appartiennent, de principes communément acceptés dans leur société ». Jean-Paul Kauffmann affirme : «...ce n'est pas la famille qui produit la familiarité, mais à l'inverse la familiarisation de l'univers proche qui produit la famille ${ }^{7}$ ». Les historiens qui, aujourd'hui, s'intéressent aux analyses de 
réseaux, pensent sensiblement la même chose. Jean-François Chauvard écrit : «Il est impossible aujourd'hui d'étudier une famille, un groupe, une communauté sans partir des individus eux-mêmes, de leur pratique, de leur comportement, de leurs relations et du même coup faire sien l'arsenal méthodologique de la micro-histoire : la reconstitution biographique, les configurations relationnelles, l'analyse de réseau ${ }^{8}$.» Lorsque l'historien "descend» au niveau de la parenté pratique, que nous définirons, avec Florence Weber, comme "l'ensemble d'obligations et de sentiments qui donne leur efficacité aux liens officiels de parenté ou qui crée d'autres liens ${ }^{9}$, l'image des relations familiales s'éclaire d'un jour nouveau. En particulier, le lien entre les frères et les sœurs prend une toute autre dimension ${ }^{10}$.

3 Le moins que l'on puisse dire, c'est que les liens adelphiques à l'époque médiévale ont été très peu étudiés ${ }^{11}$. Dans le but de répondre à la thèse de Philippe Ariès sur l'absence d'un "sentiment de l'enfance» au Moyen Âge ${ }^{12}$, les historiens des relations intrafamiliales ont longtemps mis l'accent sur les liens entre les parents et les enfants (affection, éducation). Dans une optique d'anthropologie structurale, les historiens de la parenté ont centré leur attention sur la filiation et l'alliance. Les travaux portant sur les autres périodes historiques ne sont guère plus loquaces sur les relations de germanité ${ }^{13}$.

4 Quelle situation observe-t-on dans les autres sciences sociales ? Les anthropologues ont montré la force de structuration du couple aîné-cadet dans les rapports familiaux et sociaux ou la spécificité de la relation entre un frère et une sœur dans les systèmes de parenté ${ }^{14}$. Françoise Héritier place le rapport de germanité au centre des questionnements sur les problèmes majeurs de la parenté (inceste, échange...). Elle écrit : «C'est le lieu primordial où s'expérimentent l'identité et la différence au sein d'une équivalence ${ }^{15}$. » Les psychologues ont surtout centré leur attention sur la rivalité fraternelle, l'enfant unique, les fratries dont un des membres est handicapé et sur les conséquences de la relation adelphique sur la formation de la personnalité ${ }^{16}$. Ils sont davantage intéressés par l'aspect pathogène ou les conflits à l'intérieur de la fratrie. En psychanalyse, les modes de raisonnement basés sur la triade mère-père-enfant ne se soucient que de manière latérale du lien fraternel. Les sociologues, eux aussi, évoquent les liens adelphiques dans des contextes de carences parentales ${ }^{17}$ ou (et de plus en plus) dans le cadre des familles recomposées ${ }^{18}$. Les historiens du droit abordent le sujet lorsqu'ils étudient les modes de dévolution des biens, d'une génération à une autre, l'égalité ou l'inégalité des droits lors du partage entre frères et sœurs et ont mis en lumière depuis longtemps l'importance grandissante prise par la primogéniture à partir du Moyen Âge central (le «temps du lignage»), les communautés indivisis de biens ou les frérèches.

5 Les raisons du silence des médiévistes sur ce lien de parenté ne tiennent pas à la pauvreté des sources. Il suffit d'observer les trajectoires des legs testamentaires, les principaux acteurs présents au moment des transferts patrimoniaux ou auprès des miraculés dans la documentation hagiographique, les sentiments mis en scène dans la littérature médiévale, etc., pour s'en convaincre.

6 À l'époque médiévale, les relations adelphiques méritent assurément une plus grande attention. Il faut rappeler que dans les sociétés anciennes qui connaissent une très faible espérance de vie, la coexistence entre frères et sœurs s'avère plus fréquente que celle entre parents de générations différentes ${ }^{19}$. Dans un contexte de forte mortalité, la disparition prématurée du père et de la mère ou du conjoint tend à renforcer les liens 
fraternels. Par ailleurs, la durée relativement longue du temps potentiel de fécondité d'une femme (vingt à vingt-cinq ans) et la fréquence des remariages entre conjoints d'âges très différents, produisent de forts écarts intergénésiques entraînant des différences demi-générationnelles, "obliques» (non verticales et non horizontales), venant perturber et troubler les catégories bien distinctes de "parents» ou "d'enfants ", affectant de manière sensible, les liens d'autorité et de dépendance ${ }^{20}$.

7 En outre, la société médiévale chrétienne valorise à l'extrême le lien fraternel. Par son incarnation, le Christ se présente comme le frère aîné des chrétiens. Il est, comme l'écrit saint Paul, « le premier né d'une multitude de frères » (Épître aux Romains 8, 29) ${ }^{21}$. Dans les textes hagiographiques médiévaux, frater et soror désignent souvent cette germanité spirituelle qui apparaît comme une annonce de ce que sera le Paradis céleste, après le Jugement Dernier, où tous les Élus seront réunis à Dieu, ensemble, se ressemblant tous comme des jumeaux ${ }^{22}$. Dans l'Occident chrétien, la fraternité représente la forme idéale du lien social : les chevaliers se lient par une fraternité d'armes et les moines se vivent tous comme des frères, dans un rapport d'égalité à l'égard de l'abbé, père du monastère. Dans les échanges verbaux, se donner du « Mon frère » ou du «Ma sœur » apparait toujours comme le signe d'une profonde amitié entre deux êtres.

8 Dès lors, au moment du baptême, sacrement qui intègre un nouvel être dans la communauté des chrétiens, le lien de compérage qui se noue entre le père et le parrain de l'enfant se décline fort logiquement sur le mode de la germanité. Il met les deux compères dans une position de stricte égalité l'un vis-à-vis de l'autre, créant entre eux un lien de parenté spirituelle perçu comme l'équivalent d'une relation fraternelle consanguine. Comme le fait remarquer Agnès Fine, «Si le parrainage incarne le bon amour parental, le compérage incarne le bon amour fraternel ${ }^{23}$.» Cette fraternité rituelle permet d'établir la «bonne distance », d'exclure les deux principaux dangers qui menacent les frères et sœurs, et, partant, mettent en péril la société toute entière : le fratricide et l'inceste. À partir du XIII ${ }^{\mathrm{e}}$ siècle, ce courant de fraternité religieuse s'accentue encore avec le mouvement des Ordres Mendiants dont les membres sont des « frères » et avec l'essor des confréries. Le vocabulaire qui désigne le plus fréquemment la confrérie est con(fraternitas) ou confratria. Ce type d'association propose un brassage social, offre un lieu de rencontre entre groupes sociaux différents et souvent très hiérarchisés dans la société "civile». Les statuts des confréries insistent toujours sur l'égalité entre les membres et rappellent souvent la fraternité scripturaire : "Que l'amour fraternel vous lie d'affection entre vous » (Épître aux Romains, 12, 9) ${ }^{24}$.

Le langage de la germanité déborde donc largement son champ d'application initiale, jusqu'à occuper aujourd'hui les frontons de nos mairies ${ }^{25}$. Il s'étend à d'autres formes de liens, toujours très positifs. C'est la raison pour laquelle l'emploi de «fraternel » ou de « fraternité » pose problème, le même que nous rencontrons lorsque nous qualifions de puérile l'activité d'un enfant. C'est pourquoi, nous lui préférons le terme plus neutre d' "adelphique " : adelphos, signifiant né de la même matrice. Nous pensons par ailleurs que le lexique utilisé par l'historien doit également tenir compte du sexe de la fratrie (mixte ou unisexuée). Il convient en effet de remettre en cause la très forte masculinisation des termes usités, sous couvert de neutralité : il nous parait ainsi particulièrement délicat d'utiliser «fratrie» pour désigner un ensemble de sœurs ${ }^{26}$. Nous lui préférerons le terme de « sororie ». 
10 Dans la documentation médiévale, les termes latins désignant les germains sont soror et frater, parfois flanqués de germanus ou de carnalis. Lorsque ces adjectifs n'apparaissent pas, il faut sans doute considérer ces termes dans une large acception : l'ensemble des enfants nés d'un même père et d'une même mère (siblings) mais aussi, à cause de la très forte mortalité parentale déjà évoquée, ceux qui ne partagent qu'un parent biologique ${ }^{27}$ qu'aujourd'hui nous appellerions «demi-frères" ou «demi-sœurs» (half-siblings), termes très récents, n'apparaissant sans doute pas avant le XIX ${ }^{e}$ siècle, à un moment où, pour la première fois dans l'histoire, la conjonction d'un taux de mortalité et d'un taux de divorce et de remariage très bas rend nécessaire un lexique nouveau pour désigner un phénomène rare, pourtant très répandu dans les siècles passés. Il est fort probable encore que les termes génériques de soror-frater désignent également ceux qui ne sont que des frères et sœurs sociaux, c'est-à-dire qui vivent ensemble comme des frères et sœurs (on parlerait aujourd'hui de " quart de frère » ou de "quart de sœur ») mais qui, à cause de la fréquence de la mort des parents, n'ont aucun lien de sang. Faut-il enfin élargir la définition aux «beaux-frères » et «belles-sœurs " (frères ou sœurs de son conjoint) ? Il semble, en effet, qu'en ancien français par exemple (davantage dans les termes d'adresse que de référence et peut-être davantage pour les femmes que pour les hommes), on ne fasse pas toujours la différence entre frères et sœurs germain(e)s et frères et sœurs par alliance ${ }^{28}$. Une étude reste à mener sur le vocabulaire de la germanité au sein de la parenté biologique et sociale afin d'en délimiter le champ d'application.

11 Les cinq contributions qui composent ce numéro, on l'aura compris, tentent d'éclairer non pas la position théorique d'un frère ou d'une sœur dans les systèmes de parenté mais d'étudier la relation adelphique mise en pratique dans les images et la littérature hagiographique de la fin du Moyen Âge (Chloé Maillet), les chroniques allemandes du $\mathrm{x}^{\mathrm{e}}$ siècle (Laurence Leleu), un procès de canonisation du début du XIV ${ }^{\mathrm{e}}$ siècle (Didier Lett), les archives parisiennes du Châtelet (Caroline Jeanne) ou une autobiographie allemande de la fin du $\mathrm{Xv}^{\mathrm{e}}$ siècle (Aude-Marie Certin) ${ }^{29}$.

12 Chaque auteur s'est attaché à éclairer le système des attitudes évoqué par Claude LéviStrauss et rappelé au début de cette présentation : respect et autorité du frère aîné ou de celui qui est chargé de responsabilités plus grandes au sein de la fratrie ou de l'entreprise familiale ; forte entraide lors d'un accident, d'une maladie, d'une menace ennemie ou du décès du conjoint ; rivalités feutrées des fratries associées dans une même profession ou conflits violents lors de la dévolution des biens parentaux pouvant déboucher sur des faides familiales.

13 Chaque auteur a également tenté de montrer la forte spécificité que représente le lien entre un frère et une sœur qui possède « cette caractéristique majeure au niveau de la parenté d'être à la fois une relation de l'identité et une relation de la différence absolue, alors que la relation entre germains de même sexe n'a que la première caractéristique et celle entre mari et femme la seconde ${ }^{30}$ ».

Chaque auteur, enfin, s'est soucié d'articuler la relation adelphique à d'autres liens à l'intérieur ou à l'extérieur de la parenté. Car, étudier une relation de parenté de manière empirique et en situation, permet également de montrer le caractère pluriel des individus qui, dans leur existence, sont confrontés à des expériences et des rôles variés. Occuper une position, un statut, avoir une identité, jouer un rôle de frère ou de sœur, ne signifient pas abandonner ses autres identités, rôles ou statuts au sein de la parenté (fils-fille, époux-épouse, père-mère). Ces enchevêtrements, ces superpositions 
sont rendus encore plus complexes lorsque le frère est en même temps l'associé au sein d'une même entreprise (les frères Rem décrits par Aude-Marie Certin) ou, comme dans le cas de saint Bernard, lorsque la fratrie biologique se confond avec la fratrie spirituelle (notion de transgermanité avancée par Chloé Maillet).

Face à la faiblesse du nombre d'études consacrées aux liens adelphiques, ce numéro de Médiévales se voudrait un point de départ, le début d'une aventure. La jeunesse des auteurs de ce dossier (à une forte exception près) non seulement s'inscrit dans la tradition de notre revue, mais atteste une recherche tournée vers l'avenir, visant à ajouter au sein du vaste et riche chantier de l'anthropologie de la parenté, des travaux sur la parenté pratique et sur les frères et sœurs.

\section{NOTES}

1.Comme en rend compte le récent bilan dressé par A. GUERREAU-JALABERT, R. LE JAN et J. MORSEL, « De l'histoire de la famille à l'anthropologie de la parenté », dans Les Tendances actuelles de l'histoire du Moyen Age en France et en Allemagne, J.-C. SchmitT et O. G.OeXLE dir., Paris, 2002, p. 433-447. Bien que l'utilisation du mot « famille » et ses dérivés pour désigner une « réalité » médiévale soit critiquée par les médiévistes, ardents défenseurs de l'anthropologie structurale, les auteurs de ce numéro, comme les anthropologues (par exemple : R. DELİEE, Anthropologie de la parenté, Paris, 1996, p. 15-16), continuent à s'en servir pour désigner les membres du groupe restreint (au sein de la parenté) composé de époux-épouse, père-mère, fils-fille et frère-sœur, correspondant à l'atome de parenté lévistraussien : les liens qui unissent un homme, une femme et leurs enfants... et les enfants entre-eux, Cl. LÉVI-STRAUSS, Anthropologie structurale, Paris, 1958, p. 62-65.

2..Famille et parenté dans l'Occident médiéval, G. DUBY et J. LE GOFF dir., Rome, 1977.

3..Pour un exemple d'analyse économique et sociale qui n'oublie jamais les personnes et leurs relations, voir la démarche novatrice de L. FELLER, A. GRAMAIN et F. WEBER, $L a$ Fortune de Karol. Marché de la terre et liens personnels dans les Abruzzes au haut Moyen Âge (Collection de l'Ecole française de Rome-347), Rome, 2005, en particulier (pour les conséquences sur l'étude de la parenté) le chapitre IV, p. 93-129.

4..Cl. LÉVI-STRAUSS, op. cit., p. 51 ; p. 52 pour la citation. On remarquera que, dans cette phrase, Claude Lévi-Strauss oppose (et donc dissocie) parenté et famille.

5..B. JUSSEN, « Famille et parenté. Comparaison des recherches françaises et allemandes ", dans Les Tendances actuelles... op. cit., p. 456. Cette remarque nous paraitt particulièrement pertinente à propos des nombreux travaux sur la parenté spirituelle, qui, dans sa traduction pratique, en terme de lien de parenté (parrains, marraines), joue un rôle très faible avant l'extrême fin du Moyen Âge. Pour une critique efficace de la surévaluation de la parenté au sein des sociétés, voir désormais M. GODELIER, « Nulle société n'a jamais été fondée sur la famille ou sur la parenté » dans ID., Au fondement des sociétés humaines. Ce que nous apprend l'anthropologie, Paris, 2007, p. 89-114. Si l'on adopte ces positions, on doit également critiquer le phénomène de déparentalisation observé 
par Joseph Morsel à partir du XII ${ }^{\mathrm{e}}$ siècle, J. MORSEL (avec la collaboration de C. DUCOURTIEUX), L'Histoire (du Moyen Âge) est un sport de combat... Réflexions sur les finalités de l'Histoire du Moyen Âge destinées à une société dans laquelle même les étudiants d'Histoire s'interrogent, LAMOP-Paris I, 2007, http://lamop.univ-paris1.fr/W3/JosephMorsel/ index.htm, p. 109-135.

6..M. GODELIER, Métamorphoses de la parenté, Paris, 2004, p. 93.

7..J. Cl. KAUfMANN, « La familiarité », dans Comprendre, Lien familial, 2-2001, p. 99.

8.J.-F. CHAUVARD, « Source notariale et analyse des liens sociaux. Un modèle italien ?», dans Liens sociaux et actes notariés dans le monde urbain en France et en Europe, V. GOURDON, S. BEAUVALET et F.-J. RUGGIU dir., Paris, 2004, p. 87-108 ; pour un bilan de l'engouement de la démographie historique pour l'analyse de réseaux dans la reconstitution des liens de famille, voir Histoire de la famille et analyse de réseaux, Annales de Démographie Historique, 2005-1.

9..F. WEBER, Le Sang, le Nom, le Quotidien. Une sociologie de la parenté pratique, Paris, 2005, glossaire, p. 9.

10..Dans son étude sur la parenté chez les Meo d'Inde du Nord, c'est en privilégiant la « logique des relations » et en relativisant la « logique des termes » (p. 223-224) que Raymond Jamous a pu montrer que, dans cette société, « c'est la double relation de germains 'frère' et 'sœur' qui fournit le principe structural pour déterminer la configuration de toute une série d'autres relations de parenté », R. JAMOUs, La relation frère-soeur, Parenté et rites chez les Méo de l'Inde du Nord, Paris, 1991, p. 57.

11..Pour un bilan historique récent sur les travaux des médiévistes sur cette relation, voir D. LETT, «Brothers and Sisters. New perspectives on Medieval Family History », dans Hoping for Continuity. Childhood, Education and Death in Antiquity and the Middle Ages, K. MUSTAKALLIO, J. HANSKA, H.-L. SAINIO et V. vUOLANTO dir., (Acta Instituti Romani Finlandiae-33), Rome, 2005, p. 13-23 ; on pourra consulter en particulier : F. LEDWIGE, "Relations de famille dans la correspondance de Gerson ", Revue Historique, t. CCLXXI, 1984, p. 3-23 ; B. P. MCGUIRE, « Jean Gerson and Traumas of Masculine Affectivity and Sexuality ", dans Conflicted Identities and Multiple Masculinities: Men in the Medieval West, J. MURRAY dir., New York, 1999, p. 45-72 ; I. RÉAL, Vies de saints, vie de famille. Représentation et système de la parenté dans le royaume mérovingien (481-751) d'après les sources hagiographiques, Turnhout, 2001, Partie III, chapitre 3 ; enfin, je me permets de renvoyer à mes propres travaux :D. LETT, L'Enfant des miracles. Enfance et société au Moyen Âge (XII ${ }^{e}$-XIII ${ }^{e}$ siècle), Paris, 1997, chapitre IX ; ID., "Adult brothers and juvenile uncles : Generations and age differences in families at the end of the Middle Ages ", The History of the Family , 6, 2001, p. 391-400 ; ID., " Genre et rang dans la fratrie dans les exempla de la fin du Moyen Âge (XIII $-\mathrm{XV}{ }^{\mathrm{e}}$ siècles) », dans Fratries. Frères et søeurs dans la littérature et les arts de l'Antiquité à nos jours, F. GODEAU et W. TROUBETZKoY, dir., Paris, 2003, p. 151-157 ; ID. , « La sorella maggiore 'madre sostitutiva' nei miraculi di san Luigi », dans Fratello e sorella, numéro spécial de Quaderni Storici 83, A. ARRU et S. BÆSCH GAJANO dir., a. XXVIII, n², août 1993, p. 341-353 ; ID., « Le jeune homme et les deux sœurs. Séduction et mariage dans Le Livre du chevalier de la Tour Landry pour l'enseignement de ses filles », dans La Famille, les Femmes et le Quotidien, XIV ${ }^{e}$-XVIII ${ }^{e}$ siècles, Textes offerts à Christiane KlapischZuber, I. CHABOT, J. HAYEZ et D. LETT dir., Paris, 2006, p. 333-352.

12..Ph. ARIÈs, L'Enfant et la vie familiale sous l'Ancien Régime, Paris, 1960, rééd., 1973.

13..Parmi les rares travaux dans les autres périodes, signalons Fratello e sorella, op. cit. ; L. DAVIDOFF, « Where the Stranger Begins : The Question of Siblings in Historical 
Analysis ", dans ID., Worlds Between. Historical Perspectives on Gender and Class, New York, 1995, chapitre VII, p. 206-226 (il s'agit d'une version allongée de l'article du même auteur dans Fratello e sorella... op. cit) ; Éros philadelphe, frère et sœur, passion secrète, Colloque de Cerisy, W. BANNOUR et P. BERTHIER dir. (Centre Culturel International de Cerisyla-Salle), Paris, 1992 ; J. MAîTRE, L'Orpheline de la Bérésina. Thérèse de Lisieux (1873-1897), Essai de psychanalyse socio-historique, Paris, 1995 ; V. DASEN, Jumeaux, jumelles dans l'Antiquité grecque et romaine, Kilchberg/Zürich, 2003 ; pour un ouvrage de synthèse, voir D. LETT, Histoire des frères et sœurs, Paris, 2004.

14..Les Cadets, M. SEGALEN et G. RAVIS-GIORDANI dir., Paris, 1994 ; B. VERNIER, La Genèse sociale des sentiments, aînés et cadets dans l'île grecque de Karpathos, Paris, 1991 ; R. JAMOUS, La Relation frère-sœur...op. cit., Paris, 1991 ; S. PAUWELS, « La relation frère-sœur et la temporalité dans une société d'Indonésie de l'Est », L'Homme, Revue française d'anthropologie, 116, XXX e année, Oct-Déc. 1990, pp. 7-29 ; M. XANTHAKOU, Faute d'épouses on mange des sœurs. Réalité du célibat et fantasmatique de l'inceste dans le Magne (Grèce), Paris, 1993 ; F. ZONABEND, « Mes frères, mes époux : fonctionnement de la parenté et figures de l'alliance en Basse-Normandie », dans F. HÉRITIER et E. COPET-ROUGIER dir., Les Complexités de l'alliance : les systèmes complexes d'alliance matrimoniale II, Paris, 1991, p. 206-226 ; ID., « Adopter des sœurs. Construction de la parenté et mémoire des origines ", dans L'Homme. Revue française d'anthropologie, 183, Comment être parents, 2007, p. 9-28.

15..F. HÉRITIER, Les Deux Sœurs et leur Mère. Anthropologie de l'inceste, Paris, 1995.

16.S. ANGEL, Des Frères et des sœurs. Les liens complexes de la fraternité, Paris, 1996 ; J. P. ALMODOVAR, «Penser les expériences fraternelles », dans B. CAMDESSUS dir, La Fratrie méconnue, lien du sang, lien du cœur, Paris, 1988, p. 51-70.

17..P. DURET (avec la collaboration de M. AUGUSTINI), Anthropologie de la fraternité dans les cités, Paris, 1996.

18..A. POITEVIN, Enfants de familles recomposées. Sociologie des nouveaux liens fraternels, Rennes, 2006.

19..Constat fait par Dominique Barthélemy qui voit le lien frère-sœur comme « l'axe sensible des relations de parenté », D. BARTHÉLEMY, La Société dans le comté de Vendôme de l'an mil au XIV siècle, Paris, 1993, p. 527. Cette situation s'observe au moins jusqu'à la " révolution démographique ", c'est-à-dire avant que l'Europe ne connaisse, à la fois une baisse de la mortalité puis de la fécondité, phénomène qui, en France, s'amorce dès le début du XIX ${ }^{\mathrm{e}}$ siècle ; voir par exemple, M. BAULANT, « La "famille en miettes" : sur un aspect de la démographie du XVII ${ }^{\mathrm{e}}$ siècle ", Annales E SC, juillet-octobre 1972, p. 966.

20..Sur cet aspect, je me permets de renvoyer à D. LETT, «Vieux frères et oncles jeunes : écart de générations et écarts d'âge dans les familles de la fin du Moyen Âge ", dans Lorsque l'Enfant grandit. Entre dépendance et autonomie, J.-P. BARDET, J.-N. LUC, I. ROBINROMERo et C. ROLLET, dir., Paris, 2003, p. 93-103, repris et augmenté dans « Adult brothers and juvenile uncles... ", art. cit.

21..Sur la polysémie du terme frater, voir les réflexions de saint Jérôme dans le De perpetua virginitate beatae Mariae Liber, adversus Helvidium, Liber unus, PL 23, col. 198-199. 22..Voir J. BASCHET, Le Sein du père. Abraham et la paternité dans l'Occident médiéval, Paris, 2000, en particulier, p. 168-172.

23..A. FINE, Parrains, marraines. La parenté spirituelle en Europe, Paris, 1994, p. 126 ; voir sur cet aspect, plus généralement, le chapitre IV, p. 127-163. 
24..Voir C. VINCENT, Les Confréries médiévales dans le royaume de France, XIII ${ }^{e}-\mathrm{XV}^{e}$ siècle, Paris, 1994. À l'époque moderne, le compagnonnage continue à utiliser le vocabulaire de la parenté et plus particulièrement celui de la fraternité que perpétuent les francsmaçons (les « frères trois points ») et les Poilus de 1914-1918, qui, au sortir de la Grande Guerre, dans les associations d'anciens combattants, nostalgiques et meurtris, évoqueront la « fraternité des tranchées».

25..Sur la notion de fraternité pendant la Révolution française comme sentiment, vertu, devoir et principe politique et sur les relations qu'elle entretient avec la liberté et l'égalité, voir M. DAVID, Fraternité et Révolution française, 1789-1799, Paris, 1987.

26..Certaines langues (l'italien par exemple) ne possèdent pas le mot « fratrie ", terme qui, en français, est très récent : il apparaît dans le Petit Robert vers 1970.

27..Voir infra, dans l'article de Laurence Leleu (note 4), Willigis, frater ex patre, de Thietmar de Mersbourg.

28..Voir, dans ce numéro, la contribution de Caroline Jeanne qui rappelle (note 2), qu'en ancien français, le terme de serorge, issu du latin sororius (mari de la sœur) désigne indifféremment le beau-frère ou la belle-sœur. Il est progressivement remplacé par beau-frère (première mention en 1386) et belle-sœur (attesté à partir du début du XV siècle) : article « beau » dans J. DUBOIS, H. MITTERRAND, A. DAUZAT dir., Dictionnaire étymologique et historique du français, Paris, 1998, p. 73.

29..Des interventions orales réalisées au Congrès international de Leeds les 11-14 juillet 2005 (dans le cadre de deux sessions proposées par Régine Le Jan et Didier Lett, pour le LAMOP, portant comme titre « Siblings and interchanges ») sont à l'origine de trois des cinq articles présentés dans ce numéro.

30..R. JAMOUS, La Relation frère-sœur... op. cit., p. 225.

\section{AUTEUR}

\section{DIDIER LETT}

Université Paris I-Panthéon-Sorbonne, - LAMOP, UMR 8589, 17 rue de la Sorbonne, F-75005 Paris 